\title{
ON INEQUALITIES OF THE TURÁN TYPE
}

\author{
H. SKOVGAARD
}

In 1948 Szegö [10] called attention to the following remarkable inequality of P. Turán for Legendre polynomials :

$$
\left(P_{n}(x)\right)^{2}-P_{n-1}(x) P_{n+1}(x) \geqq 0, \quad-1 \leqq x \leqq 1, \quad n \geqq 1,
$$

with equality only for $x= \pm 1$. Since that time several proofs of this inequality have been given and analogous inequalities for other functions, especially polynomials, have been established. Most of the proofs of these inequalities are based upon special recurrence relations for the particular functions. Recently Thiruvenkatachar and Nanjundiah [11] have given a rather extensive treatment of this subject and proved several inequalities of the Turán type in a simple and uniform way; they, however, also use special recurrence relations. In this connexion we may also refer to the investigations by Burchnall [2], Sansone [6], [7], and Szász [8], [9].

Of special interest is a proof given by Szegö [10], which does not use recurrence relations. It is based on an inequality deduced from a theorem of Pólya and Schur for a certain class of entire functions. By specializing this inequality almost all known inequalities of the type (0.1) may be obtained. This has already been pointed out by Szegö for some cases. In the present paper we shall deal with this subject; however, we shall take as our starting point the following more general inequality of Laguerre:

$$
\left(F^{(n)}(z)\right)^{2}-F^{(n-1)}(z) F^{(n+1)}(z) \geqq 0, \quad-\infty<z<\infty, \quad n \geqq 1 .
$$

Here $F(z)$ is an entire function of a special type. In addition we shall mention a few functions, which also satisfy an inequality of the Turán type, and two other proofs based on Laguerre's inequality.

Throughout the paper we follow the notation of G. Szegö's monograph Orthogonal polynomials (American Mathematical Society Colloquium Publications, vol. 23), New York, N. Y., 1939.

Received March 6, 1954. 
1. Let $u_{n}(x), n=0,1,2, \ldots$, be a system of real functions of a real variable $x$. The inequality

$$
\Delta_{n}(x)=\left(u_{n}(x)\right)^{2}-u_{n-1}(x) u_{n+1}(x) \geqq 0, \quad n \geqq 1,
$$

which is identical with $(0.1)$, if $u_{n}(x)=P_{n}(x),-1 \leqq x \leqq 1$, has been proved true for the following functions:

Ultraspherical (Gegenbauer) polynomials $P_{n}^{(\lambda)}(x),-1 \leqq x \leqq 1, \quad \lambda \geqq \frac{1}{2}$, Hermite polynomials $H_{n}(x), \quad-\infty<x<\infty$,

Hermite polynomials of the second kind $G_{n}(x), \quad-\infty<x<\infty$,

Laguerre polynomials

Tschebychef polynomials $L_{n}^{(\alpha)}(x),-\infty<x<\infty, \alpha \geqq 0$,

Bessel functions

Modified Bessel functions $T_{n}(x), \quad-1 \leqq x \leqq 1$,

Derivatives of Legendre polynomials $J_{n}(x), \quad-\infty<x<\infty$, $I_{n}(x), \quad-\infty<x<\infty$, $P_{n}^{\prime}(x),-\infty<x<\infty$.

Here the Hermite polynomials of the second kind have to be defined as in Appell et Kampé de Fériet, Fonctions hypergéométriques et hypersphériques, Paris, 1926, pp. 360-361. The inequality (1.1) for these polynomials has been proved by L. Koschmieder [3]. It should however be noticed that (1.1) does not hold for Hermite polynomials of the second kind defined by $G_{n}(x)=e^{-x^{2}} d^{n} e^{x^{2}} / d x^{n}$ and having $e^{2 x z+z^{2}}=\sum_{n=0}^{\infty} G_{n}(x) z^{n} / n$ ! as a generating function.

It may be added that for Bessel functions the following more general inequalities have been proved (see, in particular, [11]):

$$
\begin{array}{lll}
\left(J_{v}(x)\right)^{2}-J_{v-1}(x) J_{v+1}(x) \geqq 0, & -\infty<x<\infty, & v \geqq-1, \\
\left(I_{v}(x)\right)^{2}-I_{v-1}(x) I_{v+1}(x) \geqq 0, & -\infty<x<\infty, & v>-1 .
\end{array}
$$

For $x$ negative $J_{v-k}(x) J_{v+k}(x), \quad k=0,1, \quad$ must be interpreted as $\left(x^{2}\right)^{\nu}\left(x^{-(\nu-k)} J_{v-k}(x) x^{-(v+k)} J_{v+k}(x)\right)$. A similar interpretation should be given to $I_{v-k}(x) I_{v+k}(x)$. For $v<0$ we must exclude $x=0$.

The inequality referred to by Szegö [10] states that (1.1) holds for such values of $x$, for which the functions $u_{n}=u_{n}(x)$ have a generating function of the type

where

$$
\sum_{n=0}^{\infty} u_{n} z^{n} / n !=F(z)
$$

$$
F(z)=C e^{-\alpha z^{2}+\beta z} z^{r} \prod_{m}\left(1-z / z_{m}\right) e^{z / z_{m}}
$$


is an entire function with $\alpha \geqq 0, C, \beta$, and all $z_{m}$ real, and $\sum z_{m}{ }^{-2}$ convergent. In the following a function (1.3) is said to be of type (1.3), if these conditions are fulfilled. The proof of the inequality used by Szegö is based upon a theorem of Pólya and Schur stating that under these conditions each of the polynomials

$$
\sum_{r=0}^{n}\left(\begin{array}{l}
n \\
r
\end{array}\right) u_{r} z^{r}, \quad n=1,2, \ldots,
$$

has real zeros $z_{1}, z_{2}, \ldots, z_{n}$ only. By means of the inequality

$$
\left(\frac{z_{1}+z_{2}+\ldots+z_{n}}{n}\right)^{2} \geqq \frac{z_{1} z_{2}+z_{1} z_{3}+\ldots}{\left(\begin{array}{c}
n \\
2
\end{array}\right)}
$$

it then follows that $u_{n-1}^{2}-u_{n-2} u_{n} \geqq 0, n=2,3, \ldots$, and thus (1.1) is established.

However, the same result may be obtained by using the inequality (0.2) of Laguerre, which holds for entire functions of the type (1.3) (see [1, pp. 32-34]). To be sure, Laguerre has only considered the case where $F(z)$ is of genus 0 or 1 , that is $\alpha=0$. As indicated in Section 2, his proof may, however, easily be extended to the case $\alpha>0$. Now, assuming the functions $u_{n}=u_{n}(x), n=0,1,2, \ldots$, to have, for some values of $x$, a generating function $F(z)$ of the type (1.2)-(1.3), it follows from $u_{n}=$ $F^{(n)}(0)$, that for such $x$ inequality (1.1) is a special case of Laguerre's inequality.

2. In order to extend Laguerre's inequality to functions of type (1.3) with $\alpha>0$, we follow the method of Laguerre. Since $\sum z_{m}{ }^{-2}$ is convergent, we find by logarithmic differentiation of (1.3)

and

$$
\frac{F^{\prime}(z)}{F(z)}=-2 \alpha z+\beta+\frac{r}{z}+\Sigma\left(\frac{1}{z-z_{m}}+\frac{1}{z_{m}}\right),
$$

$$
\frac{d}{d z}\left(\frac{F^{\prime}(z)}{F^{\prime}(z)}\right)=-2 \alpha-\frac{r}{z^{2}}-\sum \frac{1}{\left(z-z_{m}\right)^{2}} .
$$

If $z$ is real and different from the zeros of $F(z)$ the expression on the right is negative or zero, hence

$$
\frac{d}{d z}\left(\frac{F^{\prime}(z)}{F(z)}\right)=\frac{F(z) F^{\prime \prime}(z)-\left(F^{\prime}(z)\right)^{2}}{(F(z))^{2}} \leqq 0,
$$

and so $(0.2)$ is true for $n=1$. Hence $(0.2)$ will be true for arbitrary $n$, 
if $F^{\prime}(z)$ and therefore all derivatives $F^{(n)}(z)$ are of the type (1.3). If $\alpha=0$ this is a simple consequence of a well-known theorem of Laguerre (see, for instance, [1, p. 32]), and if $\alpha>0$ it may be obtained essentially by copying the proof of this theorem. We meet only one difficulty, namely in proving that $F^{\prime}(z)$, written in the form (1.3), has an $\alpha \geqq 0$. However, this may be seen from the proof of another theorem of Laguerre about the derivatives of an entire function of genus $p$ and having at most a finite number of imaginary zeros (see [1, pp. 37-47]).

From the preceding it follows that if $F^{(n-1)}(z)$ is of the form

$$
F^{(n-1)}(z)=C e^{\beta z},
$$

then Laguerre's inequality becomes

$$
\left(F^{(n)}(z)\right)^{2}-F^{(n-1)}(z) F^{(n+1)}(z)=0, \quad-\infty<z<\infty .
$$

In all other cases $d\left(F^{(n)}(z) / F^{(n-1)}(z)\right) / d z<0$. This follows from an expression for this derivative analogous to (2.2). Hence equality in (0.2) occurs only at multiple zeros (if such ones exist) of $F^{(n-1)}(z)$. Consequently, if (1.2) and (1.3) are assumed to be fulfilled, equality in (1.1) holds only for such values of $x$, for which $F^{(n-1)}(z)$ is of the form $(2.4)$, or for which $z=0$ is a multiple zero of $F^{(n-1)}(z)$.

3. The inequality of Laguerre obviously holds for the following generating functions:

$$
\begin{aligned}
\sum_{n=0}^{\infty} \frac{P_{n}^{(\lambda)}(x)}{P_{n}^{(\lambda)}(1)} \frac{z^{n}}{n !}=2^{\lambda-\frac{1}{2}} \Gamma\left(\lambda+\frac{1}{2}\right) e^{x z}\left(\left(1-x^{2}\right)^{\frac{1}{2}} z\right)^{\frac{1}{2}-\lambda} J_{\lambda-\frac{1}{2}}\left(\left(1-x^{2}\right)^{\frac{1}{2}} z\right), \\
-1 \leqq x \leqq 1, \lambda>-\frac{1}{2},
\end{aligned}
$$

$$
\begin{aligned}
\sum_{n=0}^{\infty} \frac{L_{n}^{(\alpha)}(x)}{L_{n}^{(\alpha)}(0)} \frac{z^{n}}{n !}=\Gamma(\alpha+1) e^{z}(x z)^{-\frac{1}{2} \alpha} J_{\alpha}\left(2(x z)^{\frac{1}{2}}\right) & \\
& \quad-\infty<x<\infty, \alpha>-1,
\end{aligned}
$$

$$
\sum_{n=0}^{\infty} H_{n}(x) \frac{z^{n}}{n !}=e^{2 x z-z^{2}}, \quad-\infty<x<\infty
$$

$$
\sum_{n=0}^{\infty}(-1)^{n} L_{m}^{(\alpha+n)}(x) \frac{z^{n}}{n !}=e^{-z} L_{m}^{(\alpha)}(x+z), \quad-\infty<x<\infty, \quad \alpha \geqq-2,
$$

$$
\sum_{n=0}^{\infty} J_{n}(x) \frac{z^{n}}{n !}=J_{0}\left(\left(x^{2}-2 x z\right)^{\frac{1}{2}}\right), \quad-\infty<x<\infty,
$$

$$
\sum_{n=0}^{\infty}\left\{\begin{array}{c}
\cos \\
\sin
\end{array}\right\} n x \frac{z^{n}}{n !}=e^{z \cos x}\left\{\begin{array}{l}
\cos \\
\sin
\end{array}\right\}(z \sin x), \quad-\infty<x<\infty
$$


The first three of these functions are mentioned in Szegö [10]; the identity (3.4) is stated in Truesdell $[12, \mathrm{p} .84]$ while $(3.5)$ is generally known, and (3.6) is easy to prove.

The functions $u_{n}(x)$ appearing in the formulas (3.1)-(3.6) will satisfy $A_{n}(x)=0$ as follows: In (3.1) for $x= \pm 1$. In (3.2) for $x=0$. In (3.3) for no value of $x$. In (3.4) for all $x$ if $m=0$, and for $x=0$ if $\alpha=-2, n=1$. In (3.5) for $x=0$. In (3.6) for $x=p \pi$.

It may be added that, since

$$
P_{n}^{(\lambda)}(1)=\left(\begin{array}{c}
n+2 \lambda-1 \\
n
\end{array}\right), \quad L_{n}^{(\alpha)}(0)=\left(\begin{array}{c}
n+\alpha \\
n
\end{array}\right),
$$

it is easily proved that (1.1) also holds for

$$
u_{n}(x)=P_{n}^{(\lambda)}(x), \quad-1 \leqq x \leqq 1,
$$

if $\lambda \geqq \frac{1}{2}$, and, if $\alpha \geqq 0$, for

$$
u_{n}(x)=L_{n}^{(\alpha)}(x), \quad-\infty<x<\infty .
$$

Since $\cos n x$ and $\sin n x$ satisfy (1.1), the same is true for the Tschebychef polynomials of the first and second kind, $T_{n}(x)=\cos (n \operatorname{Arccos} x)$ and $U_{n}(x)=\sin [(n+1) \operatorname{Arccos} x] /\left(1-x^{2}\right)^{\frac{1}{2}}$ in the interval $-1 \leqq x \leqq 1$. That (1.1) holds for $\cos n x$ and $\sin n x$ may, of course, also be shown by direct calculation; in fact

$$
\begin{aligned}
\Delta_{n}(x) & =\cos ^{2} n x-\cos (n-1) x \cos (n+1) x \\
& =\sin ^{2} n x-\sin (n-1) x \sin (n+1) x=\sin ^{2} x \geqq 0, \quad-\infty<x<\infty .
\end{aligned}
$$

4. It has already been mentioned that the derivatives of Legendre polynomials, $P_{n}^{\prime}(x)$, satisfy the Turán inequality (1.1) for all values of $x$. This has been proved by Nanjundiah [5] by means of recurrence formulas. In this section we shall give a few other examples of derivatives satisfying (1.1).

The derivatives of the ultraspherical polynomials satisfy the recurrence formula

$$
D^{p} P_{n}^{(\lambda)}(x)=2^{p} \frac{\Gamma(\lambda+p)}{\Gamma(\lambda)} P_{n-p}^{(\lambda+p)}(x), \quad p \leqq n .
$$

Here and in the following the symbol $D^{p} f(x)$ means $d^{p} f / d x^{p}$. Since $P_{n}^{(\lambda)}(x)$ satisfies (1.1) for $-1 \leqq x \leqq 1, \lambda \geqq \frac{1}{2}$, it follows from (4.1) that $D^{p} P_{n}^{(\lambda)}(x)$ satisfies (1.1) for $-1 \leqq x \leqq 1, \lambda \geqq \frac{1}{2}-p$. Similarly, we have from the recurrence formula for Laguerre polynomials

$$
D^{p} L_{n}^{(\alpha)}(x)=(-1)^{p} L_{n-p}^{(\alpha+p)}(x),
$$


that $D^{p} L_{n}^{(\alpha)}(x)$ satisfies (1.1) for $-\infty<x<\infty, \alpha \geqq-p$. The corresponding recurrence formula for Hermite polynomials is

$$
D^{p} H_{n}(x)=2^{p} \frac{n !}{(n-p) !} H_{n-p}(x) .
$$

Since $1 \leqq p \leqq n-1$, we have

$$
(n ! /(n-p) !)^{2}>((n-1) !(n+1) !) /((n-p-1) !(n-p+1) !)
$$

hence $D^{p} H_{n}(x)$ satisfies (1.1) for $-\infty<x<\infty$, because $H_{n}(x)$ satisfies (1.1).

To prove the Turán inequality for $J_{n}^{\prime}(x)$ we first notice that the series $\Sigma_{0}^{\infty} J_{n}^{\prime}(x) z^{n} / n$ ! converges uniformly in $-\infty<x<\infty$. This follows from $2 J_{n}^{\prime}(x)=J_{n-1}(x)-J_{n+1}(x)$ and from $\left|J_{n}(x)\right| \leqq 1$. Thus, by differentiating (3.5) with respect to $x$ and using $J_{0}{ }^{\prime}(x)=-J_{1}(x)$, we get

$$
\sum_{n=0}^{\infty} J_{n}^{\prime}(x) z^{n} / n !=(z-x)\left(x^{2}-2 x z\right)^{-\frac{1}{2}} J_{1}\left(\left(x^{2}-2 x z\right)^{\frac{1}{2}}\right),-\infty<x<\infty .
$$

Since this generating function is of the type (1.3) for all $x$, the inequality (1.1) holds for $u_{n}(x)=J_{n}{ }^{\prime}(x),-\infty<x<\infty$. That (1.1) holds for $D^{p} L_{n}^{(\alpha)}(x)$ and $D^{p} H_{n}(x)$ might be proved in a similar way by using (3.2) and (3.3), respectively; it turns out, however, that the proof is more complicated in these cases, since it requires the use of asymptotic estimates for $L_{n}^{(\alpha)}(x)$ and $H_{n}(x)$ for $n \rightarrow \infty$.

5. Finally we shall mention two methods, which are similar to the method used in Section 1 in the sense that inequality (1.1) is transferred into Laguerre's inequality.

The first method consists in finding a function $F(x)$, not depending on $n$, such that $u_{n}(x)=D^{n} F(x)$. Inequality (1.1) is now satisfied for all $x$, provided $F(x)$ is of type (1.3). Thus it holds that

$$
\begin{gathered}
(-1)^{n} e^{-x^{2}} H_{n}(x)=D^{n} e^{-x^{2}}, \\
(-1)^{n} e^{-x} L_{m}^{(n)}(x)=D^{n}\left(e^{-x} L_{m}(x)\right),
\end{gathered}
$$

hence (1.1) is satisfied for the functions on the left hand side of (5.1) and (5.2) and therefore also for $H_{n}(x)$ and $L_{m}^{(n)}(x)$. The formula (5.2) appears in another connexion in Truesdell [12], which deals with functions satisfying a functional equation of the type $D_{x} F(x, v)=F(x, v+1)$. Such functions are suitable for the present purpose, because if a function $F(x, v)$ satisfies this equation for the values $v, v+1, v+2, \ldots$, of the parameter we have, $F(x, v+n)=D_{x}{ }^{n} F(x, v)$; hence $u_{n}(x)=$ 
$F(x, v+n)$ satisfies the Turán inequality (1.1), provided that $F(x)=$ $F(x, v)$ is of the type (1.3). Thus in (5.1) and (5.2) we have $v=0$, and $F(x)$ is $e^{-x^{2}}$ and $e^{-x} L_{m}(x)$, respectively.

The second method to be mentioned is used (without reference to Laguerre's inequality) by Mukherjee and Nanjundiah [4] in a proof of (1.1) for $H_{n}(x)$ and $L_{n}(x)$. In this method $u_{n}(x)$ takes the place of $F(z)$ in the inequality (2.3); this is made possible by the use of two recurrence relations and a differential equation for $u_{n}(x)$, whereby $\Delta_{n}(x)$ can be written as a product of a non-positive function and $D\left(u_{n}{ }^{\prime}(x) / u_{n}(x)\right)$. Thus Mukherjee and Nanjundiah have found the following expressions for $\Delta_{n}(x)$ :

$$
\begin{aligned}
& \Delta_{n}(x)=-\frac{1}{2 n}\left(H_{n}(x)\right)^{2} D \frac{H_{n}^{\prime}(x)}{H_{n}(x)}, \\
& \Delta_{n}(x)=-\frac{x^{2}}{n(n+1)}\left(L_{n}(x)\right)^{2} D \frac{L_{n}^{\prime}(x)}{L_{n}(x)} .
\end{aligned}
$$

We may add that the same method applies to the generalized Laguerre polynomials. Thus we find for $u_{n}(x)=L_{n}^{(\alpha)}(x) / L_{n}^{(\alpha)}(0)$, using the recurrence formulas

$u_{n-1}=u_{n}-(x / n) u_{n}{ }^{\prime}, \quad u_{n+1}=(1-x / n+\alpha+1) u_{n}+(x / n+\alpha+1) u_{n}{ }^{\prime}$ and the differential equation $x u_{n}^{\prime \prime}+(1+\alpha-x) u_{n}{ }^{\prime}+n u_{n}=0$, that

$$
\Delta_{n}(x)=-\frac{x^{2}}{n(n+\alpha+1)} u_{n}^{2} D \frac{u_{n}{ }^{\prime}}{u_{n}} .
$$

Consequently $A_{n}(x) \geqq 0$ for $\alpha>-1$, because for $\alpha>-1$ the function $L_{n}^{(\alpha)}(x)$ has real zeros only and $L_{n}^{(\alpha)}(0) \neq 0$. For $u_{n}(x)=L_{n}^{(\alpha)}(x)$ the corresponding result would be

$$
\Delta_{n}(x)=-\frac{x^{2}}{(n+1)(n+\alpha)} u_{n}^{2}\left(D \frac{u_{n}^{\prime}}{u_{n}}-\frac{\alpha}{x^{2}}\right),
$$

which shows that $\Delta_{n}(x) \geqq 0$ for $\alpha \geqq 0$.

For ultraspherical polynomials and Bessel functions the analogous representations do not immediately settle the question about the inequality (1.1).

For $u_{n}=P_{n}^{(\lambda)}(x) / P_{n}^{(\lambda)}(1)$ from the recurrence formulas $u_{n-1}=x u_{n}+\left(1-x^{2} / n\right) u_{n}^{\prime} \quad$ and $\quad u_{n+1}=x u_{n}-\left(1-x^{2} / n+2 \lambda\right) u_{n}{ }^{\prime}$ and from the differential equation 
we find

$$
\left(1-x^{2}\right) u_{n}^{\prime \prime}-(2 \lambda+1) x u_{n}^{\prime}+n(n+2 \lambda) u_{n}=0
$$

$$
\Delta_{n}(x)=-\frac{\left(1-x^{2}\right)^{2}}{n(n+2 \lambda)} u_{n}{ }^{2}\left(D \frac{u_{n}{ }^{\prime}}{u_{n}}-\frac{x}{1-x^{2}} \frac{u_{n}{ }^{\prime}}{u_{n}}\right) .
$$

Factorizing now $u_{n}=P_{n}^{(\lambda)}(x) / P_{n}^{(\lambda)}(1)$, we obtain $u_{n}{ }^{\prime} / u_{n}=\sum_{i=1}^{n}\left(x-x_{i}\right)^{-1}$, where $x_{1}, x_{2}, \ldots, x_{n}$ are the zeros of $P_{n}^{(\lambda)}(x)$, and so

$$
\Delta_{n}(x)=\frac{1-x^{2}}{n(n+2 \lambda)} u_{n}^{2} \sum_{i=1}^{n} \frac{1-x x_{i}}{\left(x-x_{i}\right)^{2}} .
$$

Since for $\lambda>-\frac{1}{2}$ each $\left|x_{i}\right|<1$, it follows that $\Delta_{n}(x) \geqq 0$ for $-1 \leqq x \leqq 1$, $\lambda>-\frac{1}{2}$.

Similarly, for $u_{v}(x)=J_{v}(x)$ from the recurrence formulas

$$
u_{v-1}=(v / x) u_{v}+u_{v}^{\prime} \quad \text { and } \quad u_{v+1}=(v / x) u_{v}-u_{\nu}{ }^{\prime}
$$

and from the differential equation

we find

$$
x^{2} u_{v}^{\prime \prime}+x u_{v}^{\prime}+\left(x^{2}-v^{2}\right) u_{v}=0
$$

$$
\Delta_{v}(x)=-\frac{1}{x} u_{\nu}^{2} D\left(x \frac{u_{v}{ }^{\prime}}{u_{v}}\right)
$$

Using the factorization

$$
J_{\nu}(x)=(x / 2)^{v} / \Gamma(v+1) \prod_{i=1}^{\infty}\left(1-x^{2} / j_{v}, i^{2}\right), \quad v \neq-1,-2, \ldots,
$$

we obtain

hence

$$
\begin{gathered}
\frac{u_{v}{ }^{\prime}}{u_{v}}=\frac{v}{x}+\sum_{i=1}^{\infty} \frac{2 x}{x^{2}-j_{v, i}^{2}} \\
D\left(x \frac{u_{v}{ }^{\prime}}{u_{v}}\right)=-4 x \sum_{i=1}^{\infty} \frac{j_{v, i}{ }^{2}}{\left(x^{2}-j_{v, i}{ }^{2}\right)^{2}}
\end{gathered}
$$

$$
\Delta_{v}(x)=4 u_{\nu}^{2} \sum_{i=1}^{\infty} \frac{j_{v, i^{2}}}{\left(x^{2}-j_{v, i}\right)^{2}} .
$$

For real $v>-1$ all the zeros of $J_{v}(x)$ are real, and therefore we have $\Delta_{v}(x)=\left(J_{v}(x)\right)^{2}-J_{v-1}(x) J_{v+1}(x) \geqq 0$ for $-\infty<x<\infty$, where $J_{v-k}(x) J_{v+k}(x)$ for negative $x$ should be interpreted as in Section 1 .

\section{REFERENCES}

1. É. Borel, Leçons sur les fonctions entières (Collection de monographies sur la théorie des fonctions), Paris, $1900 ; 2$. éd. 1921.

2. J. L. Burchnall, An algebraic property of the classical polynomials, Proc. London Math. Soc. (3) 1 (1951), 232-240. 
3. L. Koschmieder, Das Vorzeichen gewisser aus Hermiteschen Polynomen zweiter Art gebildeter Determinanten, Anz. Öster. Akad. Wiss. Math.-Nat. K1. 1951 (1951), 165-167.

4. B. N. Mukherjee and T. S. Nanjundiah, On an inequality relating to Laguerre and Hermite polynomials, Math. Student 19 (1951), 47-48.

5. T. S. Nanjundiah, $A$ note on an inequality of P. Turán for Legendre polynomials, HalfYearly J. Mysore Univ. Sect. B., N. S. 11 (1950), 57-61.

6. G. Sansone, Su una disuguaglianza di P. Turán relativa ai polinomi di Legendre, Boll. Un. Mat. Ital. (3) 4 (1949), 221-223.

7. G. Sansone, Su una disuguaglianza relativa ai polinomi di Legendre, Boll. Un. Mat. Ital. (3) 4 (1949), 339-341.

8. O. Szász, Inequalities concerning ultraspherical polynomials and Bessel functions, Proc. Amer. Math. Soc. 1 (1950), 256-267.

9. O. Szász, Identities and inequalities concerning orthogonal polynomials and Bessel functions, J. Analyse Math. 1 (1951), 116-134.

10. G. Szegö, On an inequality of P. Turán concerning Legendre polynomials, Bull. Amer. Math. Soc. 54 (1948), 401-405.

11. V. R. Thiruvenkatachar and T. S. Nanjundiah, Inequalities concerning Bessel functions and orthogonal polynomials, Proc. Indian Acad. Sci., Sect. A. 33 (1951), 373-384.

12. C. Truesdell, An essay toward a unified theory of special functions based upon the functional equation $\partial F(z, \alpha) / \partial z=F(z, \alpha+1)$ (Annals of Mathematics Studies 18), Princeton, N. J., 1948.

THE TECHNICAL UNIVERSITY OF DENMARK, COPENHAGEN 